\title{
PROPRIEDADES FÍSICAS DO SOLO, INFLUENCIADAS PELA DISTRIBUIĈ̃̃ DE POROS, DE SEIS CLASSES DE SOLOS DA REGIÃO DE LAVRAS-MĞ ${ }^{1}$
}

\author{
Soil physical properties, influenced by pores distribution, of six soil classes in the \\ region of Lavras-MG
}

\author{
Kátia Daniela Ribeiro², Stélio Maia Menezes ${ }^{3}$, Maria da Glória Bastos de Freitas Mesquita ${ }^{4}$, \\ Fabrício de Menezes Telo Sampaio ${ }^{5}$
}

\begin{abstract}
RESUMO
A distribuição de poros dos solos condiciona seu comportamento físico-hídrico. Assim, influencia os processos dinâmicos do ar e da solução do solo, bem como a potencialidade agrícola dos mesmos. Desta forma, com o presente trabalho, objetivou-se avaliar a influência da distribuição de poros sobre as propriedades físicas de seis classes de solos, sob vegetação natural, localizados na região de Lavras (MG). Coletaram-se amostras deformadas e indeformadas da camada superficial $(0-20 \mathrm{~cm})$ para a caracterização física e a determinação da curva de distribuição de poros dos solos. Os resultados indicaram que as propriedades físicas dos solos não foram influenciadas pelos poros com diâmetro entre 0,03 e $0,0375 \mathrm{~mm}$. Verificou-se, também, que os valores de macroporosidade foram diretamente proporcionais aos valores de condutividade hidráulica saturada dos solos estudados.
\end{abstract}

Termos para indexação: Porosidade do solo, tamanho dos poros do solo, condutividade hidráulica saturada, índice de vazios, grau de floculação.

\begin{abstract}
The soil pores distribution conditions its physical-hydraulic behavior. Therefore, it influences the dynamic processes of air and solution of the soil, and also its agricultural potentiality. This work aimed at evaluating the influence of pores distribution on physical properties of six classes of soil, under natural vegetation, located in the region of Lavras (MG). Disturbed and undisturbed samples were collected from superficial layer $(0-20 \mathrm{~cm})$ for physical characterization and determination of pores distribution curves. The results indicated that soil physical properties were not influenced by the pores with diameter between $0,03 \mathrm{and} 0,0375 \mathrm{~mm}$. It was also verified that macroporosity values were directly proportional to the hydraulic conductivity values of saturated soils.
\end{abstract}

Index terms: Soil porosity, soil pore size, saturated soil hydraulic conductivity, voids ratio, flocculation degree.

\section{(Recebido em 29 de agosto de 2005 e aprovado em 16 de maio de 2006)}

\section{INTRODUÇÃO}

Os poros do solo são representados por cavidades de diferentes tamanhos e formas, determinados pelo arranjamento das partículas sólidas (HILLEL, 1972; MARQUES, 2000), e constituem a fração volumétrica do solo ocupada com ar e solução (água e nutrientes). Os poros do solo correspondem, portanto, ao espaço onde ocorrem os processos dinâmicos do ar e da solução do solo (HILLEL, 1972).

A porosidade do solo interfere na aeração, condução e retenção de água, resistência à penetração e à ramificação das raízes no solo e, conseqüentemente, no aproveitamento de água e nutrientes disponíveis
(TOGNON, 1991). O solo ideal deve apresentar um volume e dimensão dos poros adequados para a entrada, movimento e retenção de água e ar para atender às necessidades das culturas (HILLEL, 1980).

O destacado papel do espaço poroso na dinâmica da água no solo foi reconhecido por vários pesquisadores, como Amaro Filho (1982) e Anderson \& Bouma (1973), que ressaltam que para se entender melhor o comportamento do solo, a distribuição dos poros por seus tamanhos é mais importante que a porosidade total. A esse respeito, Klein (1998) e Vomocil (1965) afirmam que, para diversos objetivos, tais como o movimento e armazenamento de água e gases, fluxo e retenção de calor e desenvolvimento do sistema radicular, a determinação somente da porosidade

'Parte da Dissertação de Mestrado apresentada pelo primeiro autor ao Curso de Pós-Graduação em Engenharia Agrícola, Universidade Federal de Lavras/UFLA.

${ }^{2}$ Engenheira Agrícola, MSc., Departamento de Engenharia/DEG - Universidade Federal de Lavras/UFLA - Cx. P. 3037 - $37200-000$ - Lavras, MG katiadr@bol.com.br

${ }^{3}$ Engenheiro Civil, DSc., Professor do Departamento de Engenharia/DEG - Universidade Federal de Lavras/ UFLA - Cx. P. 3037 - $37200-000$ Lavras, MG - stelio@ufla.br

${ }^{4}$ Engenheira Agrícola, DSc., Professora do Departamento de Educação/DED - Universidade Federal de Lavras/UFLA - CX. P. 3037 - $37200-000$ Lavras, MG - mgbastos@ufla.br

${ }^{5}$ Engenheiro Agrícola, MSc., Departamento de Ciência do Solo/DCS - Universidade Federal de Lavras/UFLA - CX. P. 3037 - $37200-000$ - Lavras, MG flabricio@yahoo.com.br 
total fornece informações de importância limitada. Deste modo, a determinação da distribuição dos poros na matriz do solo apresenta-se mais importante, visto que, de acordo com a distribuição dos diferentes tamanhos dos poros, pode haver restrição do fluxo de água no solo.

A presença de uma rede ideal de poros no solo, também exerce expressiva influência sobre a fertilidade do solo, afetando as relações entre drenagem, absorção de nutrientes, penetração de raízes, aeração e temperatura, relacionando-se diretamente com o desenvolvimento e produtividade das culturas (LETEY, 1985).

A análise dos poros do solo exige uma classificação dos mesmos em intervalos de tamanho distintos ( $\mathrm{S} \tilde{\mathrm{A} O}$ MATEUS, 1994). A esse respeito, muitos conceitos têm surgido no sentido de estabelecer um padrão para a classificação dos poros quanto ao tamanho, considerando a geometria e a configuração do espaço poroso (MARQUES, 2000).

Nos solos, embora não exista uma nítida separação entre poros pequenos e grandes, inúmeras classificações do diâmetro de poros são citadas na literatura, sendo que uma forma mais simplificada separa os poros em duas classes: micro e macroporos. Os microporos, também denominados poros capilares, representam os poros responsáveis pela retenção da água no solo, enquanto os macroporos representam os poros responsáveis pela drenagem e aeração do solo (BRADY, 1979).

Kiehl (1979) classifica como macro e microporos, os poros com diâmetro maior e menor, respectivamente, que $0,06 \mathrm{~mm}$, enquanto Richards (1965) utiliza diâmetro igual a $0,05 \mathrm{~mm}$ como limite de separação entre macro e microporos.

Vários autores incluem nesta classificação os mesoporos como uma classe intermediária, como Luxmoore (1981), que sugeriu uma classificação na qual os microporos apresentam diâmetro menor que $0,01 \mathrm{~mm}$; os mesoporos apresentam diâmetro entre 0,01 e 1,0 mm; e os macroporos, diâmetro maior que $1,0 \mathrm{~mm}$.

Já Klein \& Libardi (2002), classificam como macroporos, os poros com diâmetro maior que $0,05 \mathrm{~mm}$ (que perdem a água em tensões menores que $6 \mathrm{kPa}$ ), microporos, aqueles com diâmetro entre 0,05 e $0,0002 \mathrm{~mm}$ (que são esvaziados a tensões entre 6 e $1500 \mathrm{kPa}$ ) e criptoporos, poros com diâmetro menor que $0,0002 \mathrm{~mm}$ (que perdem a água apenas para tensões maiores que $1500 \mathrm{kPa})$.

Observa-se que existe uma variação muito grande na definição dos limites de diâmetro entre uma classe e outra, verificando-se que não foi estabelecida uma terminologia padrão para classificar os poros do solo quanto ao tamanho (SÃ̃ MATEUS, 1994).

A distribuição de poros do solo também pode ser avaliada considerando-se uma distribuição bimodal dos mesmos, na qual os poros do solo são classificados em poros inter-agregados e poros intra-agregados (OTHMER et al., 1991).

Os poros inter-agregados incluem os macro e mesoporos, caracterizando-se pelo fato de serem rapidamente esvaziados fazendo com que a condução de água pelo solo decresça também rapidamente. Os poros inter-agregados apresentam como função básica a aeração das raízes das plantas e a condução da água de drenagem do solo (OTHMER et al., 1991).

Os poros intra-agregados, por sua vez, correspondem aos microporos do solo e caracterizam-se por se esvaziarem mais lentamente, proporcionando uma condução de água pelo solo também mais lenta. Nos poros intra-agregados, existe uma faixa de diâmetro de poros (de $0,05 \mathrm{~mm}$ a $0,0002 \mathrm{~mm}$ ) que é a responsável pela retenção e disponibilização de água para as plantas. A água retida em poros com diâmetro menor que $0,0002 \mathrm{~mm}$ não se encontra disponível para as plantas (OTHMER et al., 1991).

A distribuição dos poros na matriz do solo desempenha papel fundamental nas relações entre as fases sólida, líquida e gasosa, determinando a evolução espacial e temporal dos processos que envolvem o movimento da água no solo. Assim sendo, a distribuição dos poros por seus tamanhos condiciona o comportamento físico-hídrico do solo, influenciando a potencialidade agrícola dos solos.

Portanto, para um conhecimento minucioso da permeabilidade do solo, dos processos de infiltração e redistribuição, da capacidade de retenção, e da extensão na qual a zona radicular é suprida com ar, a diferenciação da porosidade total de acordo com o tamanho dos poros do solo é essencial.

Neste contexto, o presente trabalho teve como objetivo avaliar a distribuição dos poros dos solos, procurando verificar sua influência sobre algumas propriedades físicas dos mesmos.

\section{MATERIAL E MÉTODOS}

Utilizaram-se amostras deformadas e indeformadas da camada superficial $(0$ a $20 \mathrm{~cm})$ de seis classes de solos (Tabela 1), sob vegetação natural, localizados na região de Lavras (MG).

A densidade do solo (Ds) foi determinada pelo método do anel volumétrico (BLAKE \& HARTGE, 1986). A densidade de partículas (Dp) foi determinada pelo método do picnômetro, segundo NBR-6508/84 da ABNT (1984a). 
TABELA 1 - Classificação e localização dos solos.

\begin{tabular}{|c|c|c|c|c|c|c|c|c|c|}
\hline \multirow[t]{2}{*}{ Solo } & \multirow[t]{2}{*}{ Classificaçãao ${ }^{(1)}$} & \multirow{2}{*}{$\begin{array}{l}\text { Localização } \\
\text { (município) }\end{array}$} & \multicolumn{2}{|c|}{$\begin{array}{c}\text { Coordenadas } \\
\text { UTM }^{(2)}\end{array}$} & \multirow{2}{*}{$\begin{array}{l}\text { Altitude } \\
\text { (m) }\end{array}$} & \multirow[t]{2}{*}{ Vegetação } & \multirow[t]{2}{*}{ Areia } & \multirow[t]{2}{*}{ Silte } & \multirow[t]{2}{*}{ Argila } \\
\hline & & & $\mathbf{X}(\mathbf{m})$ & $\mathbf{Y}(\mathbf{m})$ & & & & & \\
\hline 1 & $\begin{array}{l}\text { Neossolo } \\
\text { Quartzarênico (RQ) }\end{array}$ & Itutinga & 540.182 & 7.644 .077 & 950 & Mata nativa & 90,7 & 3,9 & 5,4 \\
\hline 2 & $\begin{array}{l}\text { Latossolo } \\
\text { Vermelho } \\
\text { distroférrico (LVdf) }\end{array}$ & Lavras & 502.970 & 7.652 .645 & 950 & Mata nativa & 32,5 & 26,5 & 41,0 \\
\hline 3 & $\begin{array}{l}\text { Latossolo } \\
\text { Vermelho-Amarelo } \\
\text { Psamítico (LVAq) }\end{array}$ & Itumirim & 526.118 & 7.646 .180 & 875 & $\begin{array}{l}\text { Pastagem } \\
\text { natural }\end{array}$ & 75,2 & 15,6 & 9,2 \\
\hline 4 & $\begin{array}{l}\text { Latossolo } \\
\text { Vermelho distrófico } \\
\text { típico (LVd) }\end{array}$ & Bom Sucesso & 516.078 & 7.670 .030 & 920 & $\begin{array}{c}\text { Mata } \\
\text { secundária }\end{array}$ & 39,8 & 23,9 & 36,3 \\
\hline 5 & $\begin{array}{l}\text { Latossolo } \\
\text { Vermelho-Amarelo } \\
\text { distrófico típico } \\
\text { (LVAd) }\end{array}$ & Nepomuceno & 485.905 & 7.653 .708 & 900 & Mata nativa. & 55,7 & 24,9 & 19,4 \\
\hline 6 & $\begin{array}{l}\text { Neossolo Flúvico } \\
\text { (RU) }\end{array}$ & Lavras & 503.662 & 7.646 .370 & 910 & Mata ciliar & 33,3 & 42,0 & 24,7 \\
\hline
\end{tabular}

(1) Dados da Embrapa.

(2) Datum SAD 69, zona 23K.

A porosidade total (PT) foi calculada a partir da relação entre a densidade do solo (Ds) e a densidade de partículas (Dp), através da equação: $\mathrm{PT}=1-\mathrm{Ds} / \mathrm{Dp}$. O índice de vazios (e) foi calculado a partir da porosidade total (PT), utilizando-se a equação: e = PT/(1-PT).

A análise granulométrica foi realizada segundo NBR-7181/84 da ABNT (1984b). A determinação da argila dispersa em água (ADA) foi realizada pelo método da pipeta (GEE \& BAUDER, 1986), com posterior cálculo do grau de floculação (GF) por meio da expressão:

$$
\mathrm{GF}=(\operatorname{argila} \text { total }-\mathrm{ADA}) /(\text { argila total })
$$

A condutividade hidráulica do solo saturado foi determinada usando-se um permeâmetro de carga constante, seguindo metodologia descrita em Klute (1965). Para calcular a condutividade hidráulica utilizou-se a equação:

$$
\mathrm{Ko}=\mathrm{Va} \cdot \mathrm{L} /[(\mathrm{A} \cdot \mathrm{t} .(\mathrm{L}+\mathrm{h})]
$$

em que:

Ko = condutividade hidráulica do solo saturado [L.T $\left.{ }^{-1}\right]$; $\mathrm{Va}=$ volume de água coletado no intervalo de tempo " $\mathrm{t}$ " $\left[\mathrm{L}^{3}\right]$; $\mathrm{L}=$ comprimento da amostra [L];
$\mathrm{A}=$ área da seção transversal da amostra $\left[\mathrm{L}^{2}\right]$;

$\mathrm{h}=$ potencial de pressão no topo da amostra [L];

$\mathrm{t}=$ tempo de coleta $[\mathrm{T}]$.

Os valores de Ko foram corrigidos para a temperatura-padrão de $20^{\circ} \mathrm{C}$, usando-se a expressão:

$$
\mathrm{Ko}_{20^{\circ} \mathrm{C}}=\mathrm{Ko}_{\mathrm{T}} * \mu_{\mathrm{T}} / \mu_{20^{\circ} \mathrm{C}}
$$

em que:

$\mathrm{Ko}_{20^{\circ} \mathrm{C}}=$ condutividade hidráulica à temperatura de $20^{\circ} \mathrm{C}$ [L.T ${ }^{-1}$;

$\mathrm{Ko}_{\mathrm{T}}=$ condutividade hidráulica à temperatura de ensaio [L.T ${ }^{-1}$;

$\mathrm{m}_{\mathrm{T}}=$ viscosidade dinâmica da água à temperatura de ensaio $\left[\mathrm{L}^{2} \cdot \mathrm{T}^{-1}\right]$;

$\mathrm{m}_{20^{\circ} \mathrm{C}}=$ viscosidade dinâmica da água à temperatura de $20^{\circ} \mathrm{C}\left[\mathrm{L}^{2} \cdot \mathrm{T}^{-1}\right]$.

A determinação da distribuição de poros do solo foi feita pelo método da dessorção de água (BOUMA, 1973), em amostras com estrutura indeformada. Foram utilizadas as tensões de 0,$02 ; 0,04 ; 0,06 ; 0,08 ; 0,1 ; 0,33$ e 15 atm. O cálculo do diâmetro dos poros do solo foi realizado através da equação:

$$
\mathrm{dv}=(4 \cdot \sigma \cdot \cos \varphi) /\left(\rho_{\mathrm{w}} \cdot \mathrm{g} \cdot \mathrm{h}\right)
$$


em que:

$\mathrm{dv}=$ diâmetro dos poros [L];

$\mathrm{s}=$ tensão superficial da água [M.T $\mathrm{T}^{-2}$;

$\mathrm{j}$ = ângulo de contato entre a água e a parede do poro;

$\mathrm{r}_{\mathrm{w}}=$ massa específica da água $\left[\mathrm{M} . \mathrm{L}^{-3}\right]$;

$\mathrm{g}=$ aceleração da gravidade $\left[\mathrm{LT}^{-2}\right] ; \mathrm{e}$

$\mathrm{h}=$ altura da coluna de água (tensão aplicada) [L].

A porcentagem de poros com diâmetro superior ao calculado para cada tensão foi obtida pela expressão:

$$
\% \mathrm{~V}=100 *\left[1-\left(\mathrm{PT}-\theta^{\prime}\right) / \mathrm{PT}\right]
$$

em que:

$\% \mathrm{~V}=$ porcentagem de poros do solo com diâmetro superior ao calculado para cada tensão;

$\mathrm{PT}=$ porosidade total do solo $(\%)$;

$\theta^{\prime}=$ umidade volumétrica correspondente à tensão utilizada para o cálculo do diâmetro dos poros, $(\%)$.

Com os dados obtidos, foram construídas as curvas de distribuição de poros (diâmetro do poro versus porcentagem de poros) para os solos estudados.

A análise estatística dos resultados obtidos consistiu na realização de análise de variância, segundo delineamento experimental inteiramente casualizado, e aplicação do teste de Scott-Knott, a $5 \%$ de probabilidade, para comparação de médias. Todas as determinações foram realizadas com quatro repetições.

\section{RESULTADOS E DISCUSSÃO}

$\mathrm{Na}$ avaliação das propriedades físicas dos solos (Tabela 2), verificou-se que o solo 4 (LVd) foi o que apresentou o maior valor de argila dispersa em água.
Os solos 1 (RQ) e 2 (LVdf) apresentaram os maiores valores de GF dentre os solos estudados.

O maior valor de densidade de partículas foi observado para o solo 2 (LVdf). O solo 6 (RU) representa um solo de várzea, que geralmente apresenta maiores teores de matéria orgânica em sua composição, justificando o menor valor de Dp encontrado para o mesmo.

$\mathrm{O}$ índice de vazios expressa a relação existente entre o volume de vazios e o volume de sólidos de um solo. $\mathrm{O}$ solo 2 (LVdf) foi o que apresentou o maior valor para o índice de vazios (o volume de vazios corresponde, aproximadamente, ao dobro da quantidade de sólidos do solo). Este maior valor encontrado para o índice de vazios permite dizer que o solo 2 (LVdf) caracteriza-se como um solo solto e poroso, justificando o menor valor de Ds encontrado para o mesmo.

A porosidade do solo é determinada pela forma como se arranjam suas partículas sólidas, destacando que se elas se arranjam em íntimo contato, ocorre predominância de sólidos na amostra de solo e a porosidade total é baixa; e se, ao contrário, as partículas se encontram arranjadas em agregados, há a predominância de vazios na amostra de solo e a porosidade é alta. Desta forma, observa-se que os menores valores de índice de vazios correspondem aos solos 1 (RQ) e 3 (LVAq) (solos arenosos), indicando que suas partículas sólidas tendem a estar arranjadas em íntimo contato, justificando os menores valores de PT e maiores valores de Ds encontrados para estes solos.

O solo 2 (LVdf) foi o que apresentou o maior valor de condutividade hidráulica saturada, devido ao menor valor de Ds e maior valor de PT encontrados para este solo, resultado de sua estrutura.

TABELA 2 - Densidade de partículas (Dp), densidade do solo (Ds), porosidade total (PT), índice de vazios (e), argila dispersa em água (ADA), grau de floculação (GF) e condutividade hidráulica saturada (Ko) dos solos.

\begin{tabular}{|c|c|c|c|c|c|c|c|}
\hline \multirow{2}{*}{ Solo } & Dp & Ds & PT & e & ADA & GF & \multirow{2}{*}{$\begin{array}{c}\text { Ko } \\
--\mathrm{cm}^{-1} \mathbf{h}^{-1}--\end{array}$} \\
\hline & \multicolumn{2}{|c|}{ 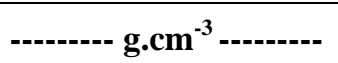 } & \multicolumn{2}{|c|}{--- $\mathbf{c m}^{3} \cdot \mathbf{c m}^{-3}$} & \multicolumn{2}{|c|}{ 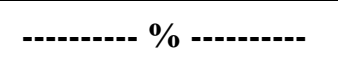 } & \\
\hline $1(\mathrm{RQ})$ & $2,616 \mathrm{c}$ & $1,454 \mathrm{a}$ & $0,4441 \mathrm{~d}$ & $0,80 \mathrm{c}$ & $0,93 \mathrm{~d}$ & 82,77 a & $42,10 \mathrm{~b}$ \\
\hline $2(\mathrm{LVdf})$ & $2,701 \mathrm{a}$ & $0,879 \mathrm{~d}$ & $0,6745 \mathrm{a}$ & $2,08 \mathrm{a}$ & $4,92 \mathrm{c}$ & $88,01 \mathrm{a}$ & 69,99 a \\
\hline 3 (LVAq) & $2,594 \mathrm{c}$ & $1,482 \mathrm{a}$ & $0,4285 \mathrm{~d}$ & $0,75 \mathrm{c}$ & $5,48 \mathrm{c}$ & $40,44 \mathrm{~b}$ & $11,99 \mathrm{c}$ \\
\hline $4(\mathrm{LVd})$ & $2,660 \mathrm{~b}$ & $1,051 \mathrm{c}$ & $0,6047 \mathrm{~b}$ & $1,54 \mathrm{~b}$ & $19,75 \mathrm{a}$ & $45,57 \mathrm{~b}$ & $33,15 \mathrm{~b}$ \\
\hline 5 (LVAd) & $2,562 \mathrm{~d}$ & $1,285 \mathrm{~b}$ & $0,4984 \mathrm{c}$ & $0,99 \mathrm{c}$ & $10,72 \mathrm{~b}$ & $44,71 \mathrm{~b}$ & $0,40 \mathrm{c}$ \\
\hline $6(\mathrm{RU})$ & $2,520 \mathrm{e}$ & $0,947 \mathrm{~d}$ & $0,6244 \mathrm{~b}$ & $1,67 \mathrm{~b}$ & $10,99 \mathrm{~b}$ & $55,48 \mathrm{~b}$ & $1,82 \mathrm{c}$ \\
\hline
\end{tabular}

Médias seguidas pela mesma letra nas colunas não diferem entre si pelo teste de Scott-Knott, a 5\% de probabilidade. 
As curvas de distribuição de poros dos solos estudados podem ser visualizadas na Figura 1. De uma maneira geral, quanto maior for a inclinação da curva de distribuição de poros do solo, maior tende a ser a diversidade do tamanho dos poros. Dessa forma, observase que o solo 1 (RQ) foi o que apresentou maior diversidade de tamanhos dos poros, uma vez que a curva de distribuição de seus poros foi a que apresentou maior inclinação.

Essa maior diversidade de tamanho de poros encontrada para o solo 1 (RQ) contraria, a princípio, a teoria clássica uma vez que se trata de um solo predominantemente arenoso. Era de se esperar que o resultado fosse o contrário, principalmente se houvesse a predominância de areia grossa em relação à areia fina. Todavia, ao analisar o fracionamento da areia para os solos estudados (Tabela 3 ), observa-se que não há predominância de areia grossa no solo 1 (RQ) e sim, de areia média e fina.

O fracionamento da areia do solo 1 (RQ) apresentase, portanto, como uma das justificativas para a maior diversidade de tamanho de poros encontrada para o mesmo. Além disso, apesar de o solo 1 (RQ) ser particularmente arenoso, a pequena quantidade de argila encontrada para o mesmo apresenta um elevado grau de floculação (Tabela 2), proporcionando a este solo certa

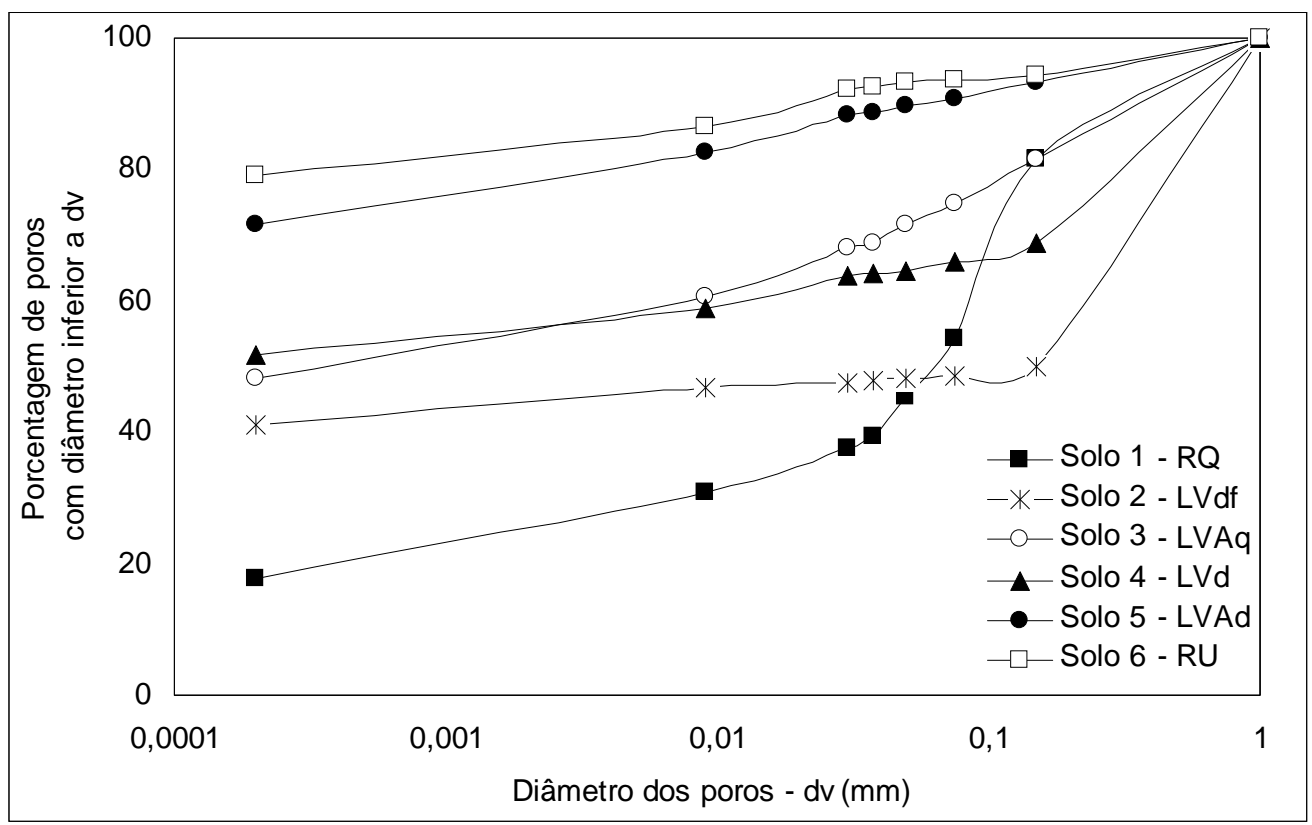

FIGURA 1 - Curvas de distribuição de poros dos solos.

TABELA 3 - Fracionamento da areia.

\begin{tabular}{|c|c|c|c|c|c|c|c|}
\hline \multirow{3}{*}{ Fração da areia } & \multirow{3}{*}{$\begin{array}{c}\text { Diâmetro } \\
\text { equivalente }\end{array}$} & \multicolumn{6}{|c|}{ Solo } \\
\hline & & 1 & 2 & 3 & 4 & 5 & 6 \\
\hline & & \multicolumn{6}{|c|}{----------- \% da areia total ------------ } \\
\hline Areia muito grossa & $2-1 \mathrm{~mm}$ & 1,03 & 20,39 & 2,39 & 4,60 & 7,05 & 1,81 \\
\hline Areia grossa & $1-0.5 \mathrm{~mm}$ & 12,83 & 22,47 & 6,85 & 14,79 & 9,79 & 2,01 \\
\hline Areia média & $0.5-0.25 \mathrm{~mm}$ & 37,40 & 13,83 & 23,13 & 27,78 & 21,29 & 6,01 \\
\hline Areia fina & $0.25-0.10 \mathrm{~mm}$ & 41,59 & 25,55 & 46,24 & 34,37 & 43,45 & 40,43 \\
\hline Areia muito fina & $0.10-0.05 \mathrm{~mm}$ & 7,15 & 17,75 & 21,39 & 18,46 & 18,41 & 49,74 \\
\hline
\end{tabular}


capacidade de agregação, o que colabora para a explicação da maior diversidade de tamanho de poros encontrada para este solo.

As curvas de distribuição de poros dos solos 5 (LVAd) e 6 (RU) apresentaram as menores inclinações, concentrando-se na parte superior da Figura 1. Isto indica uma menor variedade de tamanho de poros e reflete a predominância de poros pequenos nestes solos, o que justifica os menores valores de condutividade hidráulica saturada encontrados para os mesmos.

Os solos 1 (RQ) e 3 (LVAq), assim como solos 4 $(\mathrm{LVd})$ e $6(\mathrm{RU})$, apresentaram porosidades totais semelhantes (Tabela 2). Entretanto, analisando a Figura 1, observa-se que estes solos apresentaram curvas de distribuição de poros distintas. A diferença encontrada na distribuição de poros destes solos justifica os diferenciados valores de Ko encontrados para os mesmos, evidenciando que, no estudo do movimento da água no solo, a análise da distribuição dos poros por tamanho apresenta-se mais importante que a simples determinação da porosidade total. Isto porque a curva de distribuição de poros fornece informações que permitem diferenciar Ko e os demais atributos do solo de uma maneira mais sensível.

Na Tabela 4 são apresentados os resultados da distribuição dos poros, em intervalos de tamanho, dos solos estudados. É possível observar que os solos apresentaram uma distribuição unimodal bem definida. Tognon (1991), estudando a porosidade do solo através da porosimetria por intrusão de mercúrio detectou que a distribuição de poros era bimodal, com um primeiro máximo entre 0,005 e $0,2 \mathrm{~mm}$ de diâmetro, correspondendo aos poros inter-agregados, e um outro entre 0,00001 e $0,0001 \mathrm{~mm}$ correspondentes aos poros intra-agregados. Como neste estudo a distribuição do diâmetro dos poros foi determinada pelo método da dessorção de água, foi possível observar apenas o primeiro máximo, pois o menor diâmetro que pode ser estudado por este método é de $0,0002 \mathrm{~mm}$, correspondente à tensão de $1500 \mathrm{kPa}$.

Com relação à influência dos diferentes intervalos de tamanho de poros sobre as propriedades físicas dos solos, é possível afirmar que os poros com diâmetro entre 0,03 e $0,0375 \mathrm{~mm}$ não exerceram influência sobre as determinações dos atributos físicos apresentados na Tabela 2, uma vez que, para este intervalo de tamanho de poros, os resultados encontrados foram estatisticamente iguais para todos os solos estudados, enquanto os valores das demais propriedades físicas (Tabela 2) diferiram estatisticamente entre os solos. Conclusão similar pode ser feita para os poros com diâmetro compreendido entre os intervalos de 0,009 a $0,03 \mathrm{~mm}$ e 0,0375 a $0,05 \mathrm{~mm}$, quando somente um dos valores diferiu estatisticamente dos demais.

Os criptoporos, segundo Klein (1998), são aqueles poros nos quais a água pode permanecer retida com energia muito alta, sendo, portanto, indisponível às plantas. É a água que o solo retém quando o seu potencial matricial se encontra abaixo do ponto de murcha permanente $(<1500$ $\mathrm{kPa})$, armazenada nos poros com diâmetro inferior a

TABELA 4 - Distribuição dos poros, em intervalos de tamanho, dos solos.

\begin{tabular}{lcccccc}
\hline \multirow{2}{*}{$\begin{array}{c}\text { Diâmetro do poro } \\
(\mathbf{m m})\end{array}$} & $\mathbf{1}$ & $\mathbf{2}$ & $\mathbf{3}$ & $\mathbf{4}$ & $\mathbf{5}$ & $\mathbf{6}$ \\
\cline { 2 - 7 } & & \multicolumn{7}{c}{ Solo } \\
\cline { 2 - 7 }$>0,15$ & $0,0813 \mathrm{C}$ & $0,3362 \mathrm{~A}$ & $0,0796 \mathrm{C}$ & $0,1884 \mathrm{~B}$ & $0,0328 \mathrm{D}$ & $0,0355 \mathrm{D}$ \\
$0,15-0,075$ & $0,1212 \mathrm{~A}$ & $0,0108 \mathrm{C}$ & $0,0285 \mathrm{~B}$ & $0,0174 \mathrm{C}$ & $0,0125 \mathrm{C}$ & $0,0041 \mathrm{C}$ \\
$0,075-0,05$ & $0,0408 \mathrm{~A}$ & $0,0025 \mathrm{C}$ & $0,0139 \mathrm{~B}$ & $0,0076 \mathrm{C}$ & $0,0066 \mathrm{C}$ & $0,0028 \mathrm{C}$ \\
$0,05-0,0375$ & $0,0254 \mathrm{~A}$ & $0,0028 \mathrm{~B}$ & $0,0117 \mathrm{~B}$ & $0,0040 \mathrm{~B}$ & $0,0054 \mathrm{~B}$ & $0,0036 \mathrm{~B}$ \\
$0,0375-0,03$ & $0,0084 \mathrm{~A}$ & $0,0019 \mathrm{~A}$ & $0,0035 \mathrm{~A}$ & $0,0022 \mathrm{~A}$ & $0,0013 \mathrm{~A}$ & $0,0016 \mathrm{~A}$ \\
$0,03-0,009$ & $0,0304 \mathrm{~A}$ & $0,0056 \mathrm{~B}$ & $0,0310 \mathrm{~A}$ & $0,0301 \mathrm{~A}$ & $0,0288 \mathrm{~A}$ & $0,0367 \mathrm{~A}$ \\
$0,009-0,0002$ & $0,0576 \mathrm{~A}$ & $0,0378 \mathrm{~B}$ & $0,0536 \mathrm{~A}$ & $0,0418 \mathrm{~B}$ & $0,0547 \mathrm{~A}$ & $0,0458 \mathrm{~B}$ \\
$<0,0002$ & $0,0791 \mathrm{~F}$ & $0,2769 \mathrm{E}$ & $0,2067 \mathrm{D}$ & $0,3132 \mathrm{C}$ & $0,3562 \mathrm{~B}$ & $0,4943 \mathrm{~A}$ \\
\hline
\end{tabular}

Médias seguidas pela mesma letra nas linhas não diferem entre si pelo teste de Scott-Knott, a 5\% de probabilidade. 
0,0002 mm. Portanto, pretendendo-se utilizar os solos estudados para instalação de culturas, pode-se dizer que o solo 6 (RU) tende a apresentar os maiores problemas com relação à disponibilidade de água para as plantas, uma vez que o mesmo foi o que apresentou maior volume de criptoporos (poros com diâmetro menor que 0,0002 mm).

Os bioporos correspondem às cavidades do solo, originadas pela presença de minhocas e cupins e pelo desenvolvimento de raízes no interior do solo. Camadas superficiais do solo normalmente são dotadas de bioporos e resíduos de raízes. E, se presentes, estes grandes poros são preenchidos com água na saturação, dominando completamente o transporte de água na amostra (DIRKSEN, 1991).

No processo de amostragem do solo 2 (LVdf) foram observadas altas concentrações de raízes e intensa atividade biológica (minhocas e cupins), sendo que este solo foi o que apresentou o maior valor de Ko. Desta forma, constatase que a presença de bioporos no solo 2 (LVdf), que neste trabalho caracterizaram-se como os poros com diâmetro superior a $0,15 \mathrm{~mm}$, possibilitou que o mesmo apresentasse o maior valor de condutividade hidráulica do solo saturado.

Na Tabela 5 são apresentados os valores de macro e microporosidade dos solos estudados. Para tanto, adotou-se como limite de separação entre estas duas classes de poros o diâmetro de $0,05 \mathrm{~mm}$, valor este tido como estimativa mais adequada para efeito de física do solo (FERREIRA et al., 2003).

Os valores de microporosidade foram estatisticamente iguais somente para os solos 2 (LVdf) e 3 (LVAq). No entanto, estes dois solos apresentaram, respectivamente, o maior e o menor valor de Ko e PT. Portanto, a microporosidade dos solos apresentou-se como uma variável que, isoladamente, não foi adequada para explicar o comportamento hidráulico dos mesmos. Isto porque a geometria dos poros vai depender da estrutura de cada um dos solos.
Os solos 5 (LVAd) e 6 (RU) apresentaram os maiores valores de microporosidade, evidenciando que nestes solos a porosidade é constituída predominantemente por poros pequenos (Figura 1). Esses maiores valores de microporosidade podem ser justificados pela granulometria desses solos, que são constituídos predominantemente pelas frações areia e silte, sendo que a fração areia é composta particularmente de areia fina. Dessa forma, as partículas dos solos 5 e 6 tendem a se arranjarem de forma a estabelecerem um contato face a face, gerando uma estrutura mais adensada, na qual os grãos de areia fina e silte ocupam os espaços (“entopem”) os poros formados pela areia mais grossa, fazendo com que predominem no solo os poros pequenos (microporos).

Conforme Baver et al. (1972), um solo apresenta condições ideais de aeração para o desenvolvimento das plantas quando a macroporosidade é superior a $10 \%$. Portanto, o valor de macroporosidade para os solos 5 (LVAd) e 6 (RU) encontra-se abaixo dessas condições ideais, o que sugere que estes solos poderiam não apresentar condições satisfatórias de aeração para o desenvolvimento de eventuais culturas que pudessem vir a ser implantadas nos mesmos. Cabe ressaltar que esta observação não é válida para todas as culturas, uma vez que existem plantas que são tolerantes a níveis baixos de aeração do solo.

É possível observar que os valores de macroporosidade foram diretamente proporcionais aos valores de Ko, corroborando diversas citações da literatura, nas quais é relatado que os valores de condutividade hidráulica saturada aumentam com o incremento da macroporosidade, uma vez que esta constitui caminho preferencial ao fluxo de água no solo, sobretudo em resposta à força da gravidade (potencial gravitacional).

\section{CONCLUSÕES}

A variação na condutividade hidráulica do solo saturado, densidade do solo, porosidade total e índice de

TABELA 5 - Macro e microporosidade dos solos estudados.

\begin{tabular}{|c|c|c|c|c|c|c|}
\hline \multirow{3}{*}{ Classe de poros } & \multicolumn{6}{|c|}{ Solo } \\
\hline & 1 & 2 & 3 & 4 & 5 & 6 \\
\hline & \multicolumn{6}{|c|}{ - } \\
\hline Macroporosidade & $0,2432 \mathrm{~B}$ & $0,3494 \mathrm{~A}$ & $0,1219 \mathrm{C}$ & $0,2134 \mathrm{~B}$ & $0,0519 \mathrm{D}$ & $0,0423 \mathrm{D}$ \\
\hline Microporosidade & $0,2009 \mathrm{E}$ & $0,3250 \mathrm{D}$ & $0,3065 \mathrm{D}$ & $0,3913 \mathrm{C}$ & $0,4464 \mathrm{~B}$ & $0,5820 \mathrm{~A}$ \\
\hline
\end{tabular}

Médias seguidas pela mesma letra nas linhas não diferem entre si pelo teste de Scott-Knott, a 5\% de probabilidade. 
vazios foi explicada pela variação na distribuição do tamanho dos poros dos solos.

Os poros com diâmetro entre 0,03 e $0,0375 \mathrm{~mm}$ não explicaram bem a variação dos valores das propriedades físicas dos solos.

Os valores de condutividade hidráulica do solo saturado variaram com os valores de macroporosidade de forma diretamente proporcional.

\section{REFERÊNCIAS BIBLIOGRÁFICAS}

AMARO FILHO, J. Determinação da condutividade capilar mediante o método do perfil instantâneo. 1982. 90 f. Dissertação (Mestrado) - Universidade Federal da Paraíba, Areias, 1982.

ANDERSON, J. L.; BOUMA, J. Relationships between saturated hydraulic conductivity and morphometric data of an argillic horizon. Soil Science Society American Proceedings, Madison, v. 37, n. 3, p. 408-413, 1973.

ASSOCIAÇÃO BRASILEIRA DE NORMAS TÉCNICAS. NBR-6508/84: solo: determinação da massa específica dos grãos. Rio de Janeiro, 1984a.

ASSOCIAÇÃO BRASILEIRA DE NORMAS TÉCNICAS. NBR-7181/84: solo: análise granulométrica conjunta. Rio de Janeiro, 1984b.

BAVER, L. D.; GARDNER, W. H.; GARDNER, W. R. Soil physics. New York: J. Wiley, 1972. 498 p.

BLAKE, G. R.; HARTGE, K. H. Bulk density. In: KLUTE, A. (Ed.). Methods of soil analysis: physical and mineralogical methods. Madison: ASA-SSSA, 1986. p. 363-375.

BOUMA, J. Guide to the study of water movement in soil pedons above the watertable. Madison: University of Wisconsin, 1973. 194 p.

BRADY, N. C. Natureza e propriedades dos solos. 5. ed. São Paulo: F. Bastos, 1979. 647 p.

DIRKSEN, C. H. Unsaturated hydraulic conductivity. In: SMITH, K. A.; MULLINS, C. E. (Eds.). Soil analysis: physical methods. New York: M. Dekker, 1991. p. 209-269.

FERREIRA, M. M.; DIAS JÚNIOR, M. S.; MESQUITA, M. G. B. F.; ALVES, E. A. B. F. Física do Solo. Lavras: UFLA, 2003. 79 p.
GEE, G. W.; BAUDER, J. W. Particle size analysis. In: KLUTE, A. (Ed.). Methods of soil analysis: part 1. 2. ed. Madison: American Society of Agronomy, 1986. p. 383411.

HILLEL, D. Soil and water: physical principles and processes. 3. ed. New York: Academic, 1972. 288 p.

HILLEL, D. Fundamentals of soil physics. New York: Academic, 1980. 413 p.

KIEHL, E. J. Manual de edafologia: relações solo-planta. São Paulo: Ceres, 1979. 262 p.

KLEIN, V. A. Propriedades físico-hídrico-mecânicas de um Latossolo Roxo, sob diferentes sistemas de uso e manejo. 1998. $150 \mathrm{f}$. Tese (Doutorado) - Escola Superior de Agricultura de Luiz de Queiroz, Piracicaba, 1998.

KLEIN, V. A.; LIBARDI, P. L. Condutividade hidráulica de um Latossolo Roxo, não saturado, sob diferentes sistemas de uso e manejo. Ciência Rural, Santa Maria, v. 32, n. 6, p. 945-953, 2002.

KLUTE, A. Laboratory measurement of hydraulic conductivity of saturated soil. In: Methods of soil analysis I: physical and mineralogical properties, including statistics of measurement and sampling. Madison: American Society of Agronomy, 1965. cap. 13, p. 210-221.

LETEY, J. Relationship between soil physical properties and crop productions. Advision Soil Science, [S.1.], v. 1, p. 277-294, 1985.

LUXMOORE, R. J. Micro, meso and macroporosity of soil. Soil Science Society American Journal, Madison, v. 45, p. 671-672, 1981.

MARQUES, J. D. de O. Horizontes pedogenéticos e sua relação com camadas hidráulicas do solo. 2000. $86 \mathrm{f}$. Dissertação (Mestrado) - Escola Superior de Agricultura de Luiz de Queiroz, Piracicaba, 2000.

OTHMER, H.; DIEKKRUGER, B.; KUTILEK, M. Bimodal porosity and unsaturated hydraulic conductivity. Soil Science, Baltimore, v. 152, n. 3, p. 139-150, 1991. 
RICHARDS, L. A. Physical conditions of water in soil. In: BLACK, C. A. (Ed.). Methods of soil analysis. Madison: American Society for Testing and Materials, 1965. p. 128-152.

SÃO MATEUS, M. S. C. Determinação em laboratório da condutividade hidráulica de solos não saturados. 1994. 186 f. Dissertação (Mestrado) - Universidade de São Paulo, São Paulo, 1994.
TOGNON, A. A. Propriedades físico-hídricas do Latossolo Roxo da região de Guairá-SP sob diferentes sistemas de cultivo. 1991. 85 f. Dissertação (Mestrado) Escola Superior de Agricultura de Luiz de Queiroz, Piracicaba, 1991.

VOMOCIL, J. A. Porosity. New York: Academic, 1965. 